\title{
Dual modification of starch via partial enzymatic hydrolysis in the granular state and subsequent hydroxypropylation.
}

\begin{abstract}
The effect of enzymatic pretreatment on the degree of corn and mung bean starch derivatization by propylene oxide was investigated. The starch was enzymatically treated in the granular state with a mixture of fungal alpha-amylase and glucoamylase at 35 degrees $\mathrm{C}$ for $16 \mathrm{~h}$ and then chemically modified to produce enzyme-hydrolyzed-hydroxypropyl (HP) starch. Partial enzyme hydrolysis of starch in the granular state appeared to enhance the subsequent hydroxypropylation, as judged from the significant increase in the molar substitution. A variable degree of granule modification was obtained after enzyme hydrolysis, and one of the determinants of the modification degree appeared to be the presence of natural pores in the granules. Enzyme-hydrolyzed-HP starch exhibited significantly different functional properties compared to hydroxypropyl starch prepared from untreated (native) starch. It is evident that the dual modification of starch using this approach provides a range of functional properties that can be customized for specific applications.
\end{abstract}

Keyword: Starch; enzyme hydrolysis; Amylase; Modified starch; Hydroxypropylation. 